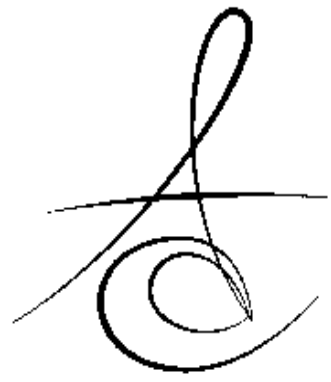

Makale Kodu/Article code: 2887

Makale Gönderilme tarihi: 10.06 .2016

Kabul Tarihi: 09.08.2016

\section{CAD/CAM SİSTEMLERİNDE MATERYAL SEÇİMİ VE KULLANIM ALANLARI}

MATERIAL SELECTIONS AND USES IN CAD/CAM SYSTEMS

Dr. Öğr. Üyesi Alper ÖZDoĞAN* öz

CAD/CAM sistemleri diş hekimliğinde büyük kolaylıklar sunmakla kalmamış çok sayıda seramik ve alt yapı materyalinin de kullanılabilmesine olanak sağlamıştır. Özellikle protetik diş tedavisinin olmazsa olmazı olan seramik materyallerinin daha hızlı ve güvenilir bir şekilde hekimlerin hizmetine sunmuştur. Günümüzde birçok seramik materyali bulunmakta ve geniş bir endikasyon yelpazesine sahip olmaktadır. $\mathrm{Bu}$ derlemede, CAD/CAM sistemlerinde materyal seçimi ve kullanım alanları anlatılmıştır.

Anahtar Kelimeler: CAD/CAM, tam seramik.

\section{ABSTRACT}

CAD / CAM systems not only provide great convenience, a plurality of ceramic material and substructure helped to that used in dentistry. Especially ceramic material, which is the sine qua non of prosthodontics is a way to provide faster and more reliable services of dentists. Today, there are many ceramic material and having a wide range of indications. In this review, material selectıons and uses in $\mathrm{CAD} / \mathrm{CAM}$ systems have been described.

Key Words: All ceramic, CAD/CAM.

\section{GİRIŞ̧}

Teknolojide meydana gelen ilerlemelere bağlı olarak diş hekimliğinde de yeni materyaller ve yüksek teknoloji içeren sistemler kullanılmaya başlanmıştır. Bu teknolojik ürünlerden birisi de bilgisayar destekli tasarım ve bilgisayar destekli üretim sistemidir. ${ }^{1,2}$

CAD (Computer Aided Design-Bilgisayar Destekli Tasarım); bir cismin bilgisayar sistemleri kullanılarak geliştirilmesi ve tasarımının yapılması anlamına gelmektedir. Bu şekilde üç boyutlu model çizimi sanal ortamda gerçekleştirilebilmektedir. ${ }^{3}$

CAM (Computer Aided Manufacturing-Bilgisayar Destekli Üretim) ise ölçülen ve planlanan veriler kullanılarak bilgisayar desteği ile üretimin yapılması anlamına gelmektedir. ${ }^{3}$

CAD/CAM sistemleri, makine destekli üretimin gerçekleştirildiği yere göre sınıflandırılmaktadır. Eğer restorasyon klinikte direkt olarak diş hazırlığı ile aynı seansta üretilirse hasta başı üretim (chair-side concept), klinikte değil de işlem laboratuvarda gerçekleşirse laboratuvarda üretim (lab-side concept), model laboratuvarda tarandıktan sonra verilerin internet yoluyla ana merkezi gönderilip alt yapı restorasyonunun ana merkezde hazırlanıp üst yapı için tekrar geri gönderildiği merkezi üretim (centralized production) olarak isimlendirilmektedir. ${ }^{4} 5$ Dental CAD/CAM sistemleri üç fonksiyonel elemandan oluşmaktadır:

1. Hazırlanan preparasyonun üç boyutlu geometrisini dijital ortama aktaran optik veya mekanik tarayıcl,

2. Elde edilecek ürüne ait verilerin oluşturulup, dental restorasyonun tasarımının yapıldığı yazıım programı,

3. Tasarlanan veriyi istenilen restorasyona dönüştüren frezeleme ünitesidir. ${ }^{5,6}$

CAD/CAM sistemlerinde restorasyonlar üretilirken değişik uygulama yöntemleri kullanılmaktadır:

\footnotetext{
*Atatürk Üniversitesi Diş Hekimliği Fakültesi Protetik Diş Tedavisi AD, Erzurum.
} 
a. Katı Bloktan Aşındırma (Eksiltme) Yöntemi: Katı bir bloktan frezler yardımıyla restorasyonların elde edildiği yöntemdir. Üretilmek istenilen restorasyon katı bloktan aşındırılarak oluşturulmaktadır. Tek üyeli restorasyonlarda oldukça başarılıdır. ${ }^{7,8}$

b. Ekleme Yöntemi: Eksiltme yöntemine alternatif olarak kullanılmaktadır. Üretim, blok kesmek yerine önceden oluşturulan rehber koordinat kullanılarak tasarlanan restorasyon tamamlanana kadar materyallerin birleştirilmesi şeklindedir. ${ }^{7}$

c. Kombine Yöntem: Ekleme ve eksiltme yöntemlerinin birlikte kullanıldı̆ı sistemdir. ${ }^{7}$

CAD/CAM sistemlerinde metal alaşımları, titanyum ve titanyum alaşımları ile geçici kompozit materyallerinin yanı sıra farklı tam seramik türleri de üretilebilmektedir.

\section{Cam Seramikler:}

\subsection{Feldspatik Seramikler:}

VITABLOCKS Mark I, Mark II, Triluxe ve RealLife (Vita Zahnfabrik, Bad Sackingen, Almanya) seramik türlerini içermektedir. ${ }^{9}$ VITABLOCKS Mark I, ilk olarak 1985 yılında üretilmiş ve inley ile onley restorasyonların yapımında kullanılmıştır. ${ }^{10}$ VITABLOCKS Mark II, inley, onley, veneer, anterior, lateral parsiyel ve full kuronların yapımında; Triluxe, onley, veneer, anterior, lateral parsiyel ve full kuronların yapımında, RealLife ise ön bölge ve veneer kuronların yapımında kullanılmaktadır. ${ }^{10}$ Materyallerin bükülme dayanımı ortalama $154 \mathrm{MPa}$, frezeleme sonrası ağza tatbik edilebilir, üstün translusent ve kolay cilalanabilme özelliklerine sahiptir. ${ }^{11}$

\subsection{Lösit ile Güçlendirilmiş Seramikler:}

1988 yılında ProCAD (Ivoclar Vivadent; Schaan, Liechtenstein) CEREC (Sirona Dental Systems; Bensheim, Almanya) ile kullanılmak üzere geliştirilmiştir. Özellikleri Empress (Ivoclar Vivadent; Schaan, Liechtenstein) sistemine benzemektedir ve lösit ile güçlendirilmiş cam seramiktir. ${ }^{12} 2006$ yılında Empress CAD sistemi ProCAD'in devamı olarak geliştirilmiş olup esas farklılık üretim aşamasında 1-5 $\mu \mathrm{m}$ paritkül boyutundaki \% 45 oranında lösit içeriğidir. ${ }^{13}$ Materyalin bükülme dayanımı 160 MPa'dır. Klinik olarak tek diş restorasyonları için yüksek, düşük translusensi ve polikromatik bloklar şeklindedir. Bu gruptaki diğer bir materyal grubu ise Paradigm C (3M ESPE; Seefeld, Almanya)'dir.

\subsection{Lityum Disilikat ile Güçlendirilmiş} Seramikler:

Lityum disilikat ile güçlendirilmiş seramikler yaklaşık 350-450 MPa bükülme dayanımı değerleri ile lösit ile güçlendirilmiş seramiklerden daha dayanıklıdır. ${ }^{14} 2006$ yılında üretilmiş olan e.max CAD (Ivoclar Vivadent; Schaan, Liechtenstein) chair-side monolitik bir restoratif materyaldir. IPS e.max CAD bloklar, kuartz, lityum dioksit, fosfor oksit, alümina, potasyum oksit ve diğer bileşenlerden oluşmaktadır. Doğal parlaklık, translusenslik ve geniş renk aralığına sahiptir. Teleskop kuron, full kuron, inley, onley, ön ve arka bölge kuronlar, implant üstü kuronların yapımında kullanılmaktadır (Tablo 1). ${ }^{15}$

Tablo 1. Cam seramikler ve kullanım alanları

\begin{tabular}{|l|c|c|c|}
\hline \multirow{4}{*}{$\begin{array}{l}\text { Feldspatik } \\
\text { Seramikler }\end{array}$} & Materyal & $\begin{array}{c}\text { Üretici } \\
\text { Firma }\end{array}$ & Kullanım Alanı \\
\cline { 2 - 4 } & $\begin{array}{c}\text { Vitablocks } \\
\text { Mark I }\end{array}$ & $\begin{array}{c}\text { Vita } \\
\text { Vitablocks } \\
\text { Mark II }\end{array}$ & $\begin{array}{c}\text { Inley ve onley } \\
\text { restorasyonlar }\end{array}$ \\
\cline { 2 - 4 } & Vealla & $\begin{array}{c}\text { Inley, onley, } \\
\text { veneer, anterior, } \\
\text { lateral parsiyel ve } \\
\text { full kuronlar }\end{array}$ \\
\hline $\begin{array}{l}\text { Lösit ile } \\
\text { Güçlendirilmiş } \\
\text { Seramikler }\end{array}$ & ProCAD & Ivoclar & $\begin{array}{c}\text { Ön bölge ve veneer } \\
\text { kuronlar }\end{array}$ \\
\cline { 2 - 4 } $\begin{array}{c}\text { Eek diş } \\
\text { restorasyonlar }\end{array}$ \\
\hline $\begin{array}{l}\text { Lityum Disilikat } \\
\text { ile } \\
\text { Güçlendirilmiş } \\
\text { Seramikler }\end{array}$ & $\begin{array}{c}\text { CAD } \\
\text { CADax }\end{array}$ & Ivoclar & $\begin{array}{c}\text { Tek diş } \\
\text { restorasyonlar }\end{array}$ \\
\hline
\end{tabular}

2. Alümina veya Zirkonya ile Güçlendirilmiş Seramikler:

2.1. Cam İnfiltre CAD/CAM Seramikler:

Alümina ve zirkonya esaslı seramikler 1993 yılından beri CAD/CAM ile üretilmektedir. InCeram porselen grubu (InCeram Alumina, Spinell and Zirconia; Vita Zahnfabrik, Bad Sackingen, Almanya) slip cast ve cam infiltrasyonu yöntemi ile üretilen sistemlerdir. ${ }^{12}$ Bloklar seramik tozunun açık gözenekli mikro yapıya ulaşana kadar sıkıştırılması yöntemi olan kuru presleme (dry pressing) ile üretilmekte ve daha sonra sinterize edilip cam yapıya infiltre olmaktadır. ${ }^{16}$

InCeram Alumina, Spinell ve Zirconia'nın bükülme dayanımları sırasıyla 450-600 MPa, $350 \mathrm{MPa}$, 700 MPa'dır. $^{11}$ CAD/CAM InCeram Spinell bu grup içinde özellikle ön bölge kuronları için en translusens materyal olup, 5 yıllık bir takip sonucu başarı oranının \% 91.7 olduğu bildirilmiştir. ${ }^{17,}{ }^{18}$ CAD/CAM InCeram Alumina tek diş ön ve arka bölge kuronlarının yapımı 
için endikedir, premolar ve molar kuronlara CEREC (Sirona, Beinsheim, Almanya) sistemi ile yapılan bir çalışmanın 5 yıllık takibi sonucunda başarı oranının \% 92 olduğu belirtilmiştir. ${ }^{18}$ CAD/CAM InCeram Zirconia, cam infiltre edilmiş zirkonyanın alümina ile güçlendirilmiş şekli olup bu grubun en dayanıkı materyaldir. ${ }^{19}$ Ancak zirkonyanın opak karakteri bu sistemin sadece kuron alt yapısı ve arka bölgedeki köprülerde kullanım alanı bulmasına sebep olmuştur. ${ }^{20}$

\subsection{Polikristalin CAD/CAM Seramikler:}

Polikristalin yapıda olan alümina ve zirkonya seramikleri ise cam matrikse herhangi bir işlem yapılmadan yoğun normal diziler halinde paketlenir ve daha sonra sinterlenir. ${ }^{13,} 21$ Ortaya çıkan yoğun kristal fazla birlikte çatlak ilerlemesi azalmış ve üstün mekanik özelliklere sahip olunmuştur. Ancak bu dayanıkılık artışına bağlı olarak bu tür protezler CAD/CAM sistemi olmadan üretilemezler. ${ }^{21} \mathrm{Bu}$ tür materyaller sicak izostatik presleme (hot isostatic pressing) yöntemi ile sinterize edilmektedir. ${ }^{22} \mathrm{Bu}$ yöntemde seramik tozu içine alınmış bir kapsül sistemine yüksek izostatik basınç uygulanmakta, sinterleme sırasında yüksek kuvvet korunmakta ve seramik blok gerçek boyutlarına ulaşmaktadır. Bu blokların frezeleme işlemi katı işleme (hard machining) olarak da isimlendirilmektedir. ${ }^{23}$

Procera AllCeram (Nobel Biocare, Göteborg, İsveç), 1993 yllında üretilen ilk tam yoğun polikristalin seramik olup \% 99 oranında Alümina içerir ve bükülme dayanımı 600 MPa'dır. ${ }^{13,} 24-26$ Procera AllCeram polikristalin bir seramik olduğu için opaktır ve translusenslik bakımından aynı kalınlıktaki tam seramik materyalleri ile karşılaştıııldığında Empress ve Empress II sistemlerinin arasında yer almaktadır. Bu nedenle ön dişlerinde renklenme olmayan hastalarda laminate yapımı için kullanımı önerilmektedir. ${ }^{27,} 28$ Ön ve arka bölgedeki tek kuronlarda ise 5 yıllık başarı oranının \% 97, 10 yıllık başarı oranının ise \% 93.5 olduğu bildirilmiştir. ${ }^{29}$, 30 Procera AllCeram sabit bölümlü protezlerde de kullanılabilmektedir. ${ }^{31}$

Zirkonya polimorfik bir malzeme olup, monoklinik, tetragonal ve kübik fazlardan oluşmaktadır. ${ }^{23,} 32$ Zirkonyuma ilave edilen yttrium oksit $\left(\mathrm{Y}_{2} \mathrm{O}_{3}\right)$ yapıyı oda sıcaklığında stabilize etmektedir. ${ }^{33}$ Zirkonyumun bükülme dayanımı 900-1200 MPa arasında olup alüminanın yaklaşık iki katıdır. ${ }^{34,} 35$ Zirkonyum, diş hekimliğinde pek çok alanda kullanılmaktadır; ortodontik braketler, endodontik postlar, kuronlar, sabit bölümlü protezler, implantlar ve implant abutmentleri gibi. ${ }^{36-39}$ Günümüzde zirkonyum için birçok CAD/CAM sistemi bulunmakta ve bloklardan freze edilerek kullanılmaktadır: Lava (3M Espe Dental AG, Seefeld,Almanya), Cercon (DeguDent, Hanau, Almanya), Procera Zirconia (Nobel Biocare AB, Goteborg, İsveç), e.max ZirCAD (Ivoclar Vivadent; Schaan, Liechtenstein)(Tablo 2). ${ }^{12}$

Tablo 2. Alumina ve zirkonya ile güçlendirilmiş seramikler ve kullanım alanları

\begin{tabular}{|c|c|c|c|}
\hline & Materyal & $\begin{array}{l}\text { Üretici } \\
\text { Firma }\end{array}$ & Kullanım Alanı \\
\hline \multirow{3}{*}{$\begin{array}{l}\text { Cam İnfiltre } \\
\text { Seramikler }\end{array}$} & $\begin{array}{l}\text { InCeram } \\
\text { Spinell }\end{array}$ & Vita & Ön bölge kuronlar \\
\hline & $\begin{array}{l}\text { InCeram } \\
\text { Alumina }\end{array}$ & Vita & $\begin{array}{l}\text { Tek diş ön ve arka bölge } \\
\text { kuronlar }\end{array}$ \\
\hline & $\begin{array}{l}\text { InCeram } \\
\text { Zirconia } \\
\end{array}$ & Vita & $\begin{array}{l}\text { Kuron alt yapısı ve arka } \\
\text { bölge köprüler }\end{array}$ \\
\hline $\begin{array}{l}\text { Polikristalin } \\
\text { Seramikler }\end{array}$ & $\begin{array}{l}\text { Procera } \\
\text { AllCeram }\end{array}$ & $\begin{array}{l}\text { Nobel } \\
\text { Biocare }\end{array}$ & $\begin{array}{l}\text { Laminate yapımı, ön ve } \\
\text { arka bölge tek kuronlar }\end{array}$ \\
\hline \multirow{4}{*}{$\begin{array}{l}\text { Zirkonya } \\
\text { Seramikler }\end{array}$} & Lava & 3M Espe & \multirow{4}{*}{$\begin{array}{l}\text { Ön ve arka bölge kuron } \\
\text { köprüler, implant, } \\
\text { implant abutment, } \\
\text { endodontik } \\
\text { ortodontik braket. post, }\end{array}$} \\
\hline & $\begin{array}{l}\text { Procera } \\
\text { Zirconia } \\
\end{array}$ & $\begin{array}{l}\text { Nobel } \\
\text { Biocare }\end{array}$ & \\
\hline & $\begin{array}{l}\text { e.max } \\
\text { ZíRCAD }\end{array}$ & Ivoclar & \\
\hline & Cercon & Degudent & \\
\hline
\end{tabular}

\section{Geçici Restorasyonlar:}

Rezin materyaller, kazıma ünitelerinde kayıp mum (lost wax) tekniğinde kullanılabildiği gibi, kısa ve uzun dönem geçici restorasyonlarda da kullanılabilmektedir (Tablo 3). ${ }^{40}$

Tablo 3. Rezin materyaller ve kullanım alanları

\begin{tabular}{|l|l|l|}
\hline $\begin{array}{l}\text { Rezin } \\
\text { Materyal }\end{array}$ & $\begin{array}{l}\text { Üretici } \\
\text { Firma }\end{array}$ & Kullanım Alanı \\
\hline CAD-Waxx & Vita & $\begin{array}{l}\text { Kayıp mum tekniği için serbest akrilik } \\
\text { polimeri }\end{array}$ \\
\hline $\begin{array}{l}\text { Cercon } \\
\text { base cast }\end{array}$ & Degudent & $\begin{array}{l}\text { Kayıp mum tekniği için artıksız } \\
\text { yakılabilen reçine }\end{array}$ \\
\hline $\begin{array}{l}\text { Everest C- } \\
\text { cast }\end{array}$ & Kavo & $\begin{array}{l}\text { Kayıp mum tekniği için artıksız } \\
\text { yakılabilen reçine }\end{array}$ \\
\hline $\begin{array}{l}\text { CAD-Temp } \\
\text { Block }\end{array}$ & Vita & $\begin{array}{l}\text { Uzun dönem geçici full ve parsiyel } \\
\text { kuronlar ve sabit hareketli protezlerde } \\
\text { 2 veya daha fazla pontik yapımı için } \\
\text { mikrofil akrilik polimer }\end{array}$ \\
\hline $\begin{array}{l}\text { Everest C- } \\
\text { Temp }\end{array}$ & Kavo & $\begin{array}{l}\text { Uzun dönem geçici kuronlar ve sabit } \\
\text { bölümlü ilave } \\
\text { veneerlenmesi protezlerin } \\
\text { güçlendirilmiş polimer }\end{array}$ \\
\hline $\begin{array}{l}\text { Artegral } \\
\text { imCrown }\end{array}$ & Merz & $\begin{array}{l}\text { Uzun dönem ön bölge kişisel geçici } \\
\text { kuron yapımı }\end{array}$ \\
\hline Telio CAD & Ivoclar & Sabit protezlerde geçici kuron yapımı \\
\hline
\end{tabular}

Vita CAD-Temp (Vita Zahnfabrik, Bad Sackingen, Almanya) sabit protezlerde kullanılan 40-55 $\mathrm{mm}$ uzunluğunda hazırlanabilen yüksek çapraz bağlantılı mikrofil polimerlerdir. Dört farkı tonu veya dört farklı renk katmanına sahip olan tek blok şeklinde 
üretilmektedir. ${ }^{41}$ Telio CAD (Ivoclar Vivadent; Schaan, Liechtenstein), sabit protezlerin geçici kuronlarının yapımı için üretilen çapraz bağlantılı polimetil metakrilat bloklardır. Bu bloklar, kendi kendine sertleşen (self-curing) kompozit rezin, hassasiyet giderici ile simandan oluşan Telio sisteminin bir parçasıdır ve beş farklı renk tonuna sahiptir. ${ }^{42}$ Her iki geçici materyali için de üretici firmalar tarafından 1 yıllık klinik uygulama süresi tavsiye edilmektedir. ${ }^{43}$

\section{SONUÇ}

Diş hekimliği kliniklerine her geçen gün yeni teknolojik ürün ve materyaller girmektedir. CAD/CAM sistemleri de bu ürünlerden birisi olup, zaman tasarrufu, hata oranının minimuma inmesi gibi hekim ve hastalara büyük avantajlar sağlamaktadır. Protetik tedaviler için de çok sayıda seramik materyali bulunmakta ve vakanın çeşitliliğine göre de bu materyaller CAD/CAM sistemleri yardımıyla kullanılabilmektedir.

Alper Özdoğan: ORCID ID: 0000-0003-0649-3056 Funda Bayındır: ORCID ID: 0000-0001-5699-2879

\section{KAYNAKLAR}

1. Kalaycı BB, Bayındır F. Güncel dental bilgisayar destekli tasarım/bilgisayar destekli üretim sistemleri, Atatürk Üniv.Diş Hek Fak Derg 2015; 11:129-36.

2. McMillian P. Glass-Ceramics. 2. edition. London; Academic Press. 1979.

3. Jedynakiewicz NM, Martin N. CEREC: science, research, and clinical application. Compend Contin Educ Dent 2001; 22: 7-13.

4. Burke EJ, Qualtrough AJ. Aesthetic inlays: composite or ceramic? Br Dent J 1994; 176: 53-60.

5. Hickel R, Dasch W, Mehl A, Kremers L. CAD/CAM-fillings of the future? Int Dent J, 1997, 47: 247-58.

6. Mehl A, Hickel R. A new optical 3D-scanning system for CAD/CAM technology. Int J Comput Dent 1999; 2: 129-36.

7. Strub JR, Rekow ED, Witkowski S. Computer-aided design and fabrication of dental restorations: current systems and future possibilities. J Am Dent Assoc 2006; 137: 1289-96.

8. Manicone PF, Rossi Iommetti P, Raffaelli L. An overview of zirconia ceramics: basic properties and clinical applications. J Dent 2007; 35: 819-26.
9. Mormann WH, Bindl A. All-ceramic, chair-side computer-aided design/computer-aided machining restorations. Dent Clin North Am 2002; 46: 405-26.

10. Otto T, De Nisco S. Computer-aided direct ceramic restorations: a 10-year prospective clinical study of Cerec CAD/CAM inlays and onlays. Int J Prosthodont 2002; 15: 122-8.

11. Giordano R. Materials for chairside CAD/CAMproduced restorations. J Am Dent Assoc 2006; 137 Suppl: 14s-21s.

12. Li RW, Chow TW, Matinlinna JP. Ceramic dental biomaterials and CAD/CAM technology: state of the art. J Prosthodont Res 2014; 58: 208-16.

13. Giordano R, McLaren EA. Ceramics overview: classification by microstructure and processing methods. Compend Contin Educ Dent 2010; 31: 682-4, 686, 688 passim; quiz 698, 700.

14. Ho GW, Matinlinna JP. Insights on Ceramics as Dental Materials. Part II: Chemical Surface Treatments. Silicon 2011; 3: 117-123.

15. Tysowsky GW. The science behind lithium disilicate: a metal-free alternative. Dent Today 2009; 28: 112-3.

16. Apholt W, Bindl A, Luthy $H$, Mormann WH. Flexural strength of Cerec 2 machined and jointed InCeramAlumina and InCeram-Zirconia bars. Dent Mater 2001; 17: 260-7.

17. Bindl A, Mormann WH. Survival rate of monoceramic and ceramic-core CAD/CAM-generated anterior crowns over 2-5 years. Eur J Oral Sci, 2004; 112: 197-204.

18. Bindl A, Mormann WH. An up to 5-year clinical evaluation of posterior in-ceram CAD/CAM core crowns. Int J Prosthodont 2002; 15: 451-6.

19. Chong KH, Chai J, Takahashi Y, Wozniak W. Flexural strength of In-Ceram alumina and InCeram zirconia core materials. Int J Prosthodont 2002; 15: 183-8.

20. Heffernan MJ, Aquilino SA, Diaz-Arnold AM, Haselton DR, Stanford CM, Vargas MA. Relative translucency of six all-ceramic systems. Part II: core and veneer materials. J Prosthet Dent 2002; 88: $10-5$.

21. Kelly JR, Benetti P. Ceramic materials in dentistry: historical evolution and current practice. Aust Dent ] 2011; 56 Suppl 1: 84-96. 
22. Li J, Liao H, Hermansson L. Sintering of partiallystabilized zirconia and partially-stabilized zirconiahydroxyapatite composites by hot isostatic pressing and pressureless sintering. Biomaterials 1996; 17: 1787-90.

23. Miyazaki T, Nakamura $T$, Matsumura $H$, Ban $S$, Kobayashi T. Current status of zirconia restoration. J Prosthodont Res 2013; 57: 236-61.

24. Andersson $M$, Oden A. A new all-ceramic crown. A dense-sintered, high-purity alumina coping with porcelain. Acta Odontol Scand 1993; 51: 59-64.

25. Zeng K, Oden A, Rowcliffe D. Flexure tests on dental ceramics. Int J Prosthodont, 1996, 9: 434-9.

26. Brunton PA, Smith P, McCord JF, Wilson NH. Procera all-ceramic crowns: a new approach to an old problem? Br Dent J 1999; 186: 430-4.

27. Chu FC, Andersson B, Deng FL, Chow TW. Making porcelain veneers with the Procera AllCeram system: case studies. Dent Update 2003; 30: 454$8,460$.

28. Hager B, Oden A, Andersson B, Andersson L. Procera AllCeram laminates: a clinical report. J Prosthet Dent 2001; 85: 231-2.

29. Odman P, Andersson B. Procera AllCeram crowns followed for 5 to 10.5 years: a prospective clinical study. Int J Prosthodont 2001; 14: 504-9.

30. Fradeani $M$, D'Amelio $M$, Redemagni $M$, Corrado $M$. Five-year follow-up with Procera all-ceramic crowns. Quintessence Int, 2005; 36: 105-13.

31. Raigrodski AJ. Contemporary all-ceramic fixed partial dentures: a review. Dent Clin North Am 2004; 48: 531-44.

32. Liu PR, Essig ME. Panorama of dental CAD/CAM restorative systems. Compend Contin Educ Dent 2008; 29: 482, 484, 486-8 passim.

33. Catledge SA, Cook M, Vohra YK, Santos EM, McClenny MD, David Moore K. Surface crystalline phases and nanoindentation hardness of explanted zirconia femoral heads. J Mater Sci Mater Med 2003; 14: 863-7.

34. Christel P, Meunier A, Heller M, Torre JP, Peille CN. Mechanical properties and short-term in-vivo evaluation of yttrium-oxide-partially-stabilized zirconia. J Biomed Mater Res 1989; 23: 45-61.

35. Chai J, Chu FC, Chow TW, Liang BM. Chemical solubility and flexural strength of zirconia-based ceramics. Int J Prosthodont 2007; 20: 587-95.
36. Springate SD, Winchester LJ. An evaluation of zirconium oxide brackets: a preliminary laboratory and clinical report. Br J Orthod, 1991, 18: 203-9.

37. Meyenberg $\mathrm{KH}$, Luthy $\mathrm{H}$, Scharer P. Zirconia posts: a new all-ceramic concept for nonvital abutment teeth. J Esthet Dent 1995; 7: 73-80.

38. Luthardt RG, Sandkuhl O, Reitz B. Zirconia-TZP and alumina--advanced technologies for the manufacturing of single crowns. Eur J Prosthodont Restor Dent 1999; 7: 113-9.

39. Kohal RJ, Klaus G, Strub JR. Zirconia-implantsupported all-ceramic crowns withstand long-term load: a pilot investigation. Clin Oral Implants Res 2006; 17: 565-71.

40. Beuer F, Schweiger J, Edelhoff D. Digital dentistry: an overview of recent developments for CAD/CAM generated restorations. Br Dent J 2008; 204: 50511.

41. Vita machinable polymers: produn information. Vita Zahnfabrik 2010.

42. Telio CS and Telio-CAD. Instructions for Use. Ivoclar Vivadent Technical. December 2009.

43. Fasbinder DJ. Materials for chairside CAD/CAM restorations. Compend Contin Educ Dent 2010; 31: 702-4, 706, 708-9.

\author{
Yazışma Adresi \\ Dr. Öğr. Üyesi Alper ÖZDOĞAN \\ Atatürk Üniversitesi \\ Diş Hekimliği Fakültesi, \\ Protetik Diş Tedavisi AD \\ Erzurum \\ TIf; 5392018440 \\ e-mail: alprozdgn@gmail.com
}

